\title{
Computational and experimental studies for probe ring radius in MIMO OTA test systems
}

\author{
Dmytro Anin \\ Centre for Wireless Communications \\ University of Oulu \\ Erkki Koiso-Kanttilan katu 3, 90570 Oulu, \\ Finland \\ Email: Dmytro.Anin@oulu.fi
}

\author{
Miah Md. Suzan \\ Department of Electronics \\ and Nanoengineering \\ Aalto University \\ Otakaari 5, 02150 Espoo, \\ Finland \\ Email:md.miah@aalto.fi
}

\author{
Erkki Salonen \\ Centre for Wireless Communications \\ University of Oulu \\ Erkki Koiso-Kanttilan katu 3, 90570 Oulu, \\ Finland \\ Erkki.Salonen@oulu.fi
}

\begin{abstract}
The effect of the probe ring size in OTA test systems was studied based on simulations and OTA measurements in the anechoic chamber of the University of Oulu. The results are presented for two methodologies: Prefaded Synthesis (PFS) and Plane Wave Synthesis (PWS). Each size and synthesis type was validated for UMI and Uma radio channel models and for the cases the DUT in free space and in phantom hand.
\end{abstract}

\section{INTRODUCTION}

This work is dedicated to experimental and theoretical study of measurements methods using multiple-output (MIMO) technique over-the-air (OTA). MIMO is nowadays technique that is widely used in wireless communication since it has such advantages as improvement of connection performance and it provides better spectrum efficiency. OTA tests is the most realistic test methods that provide the most accurate wireless link characterization since they emulate realistic for wireless link scenario when data is transferred via radiomodule and antennas and currently widely used for multi-antenna equipped mobile devices [1]-[3]. OTA test standardization for multiple-input and multiple-output (MIMO) terminals is coordinated by Cellular Telecommunications Industry Association (CTIA) and 3rd Generation Partnership Project (3GPP) [4]. Such test measurements are usually performed in anechoic and reverberation chambers [5], [6]. There is an increasing industry demand to reduce the size of the anechoic chamber as a base of MIMO-OTA test setup. Possible anechoic chamber sizes were studied theoretically in for instance [7], [8]. Therefore real measurements are crucial in validation of theoretical predictions and focus of current experimental and theoretical study is physical dimensions of anechoic chamber.

This article is structured in the following way: in Section II theoretically modeled OTA ring size effect and findings are reported. In Section III simulations and measurements are discussed and computations are compared to experimental measurements. Section IV consists of conclusions of the paper.

\section{STUdiES OF PROBE RING RADIUS}

Typical measurement requires creation of certain electromagnetic environment inside a test area of the chamber. To do that anechoic chamber is equipped with evenly angularly distributed multiple probe antennas that belong to same imaginary plane. The area inside this imaginary ring formed by probe antennas is called a OTA ring and schematically is shown in Fig. 1. Device to be tested or device under test (DUT) is placed in the center of the circle of probe antennas pointed towards its imaginary center is used to create signals from desired directions, therefore provides possibility to emulate direct, reflected and interfered links, etc. In these studies Nokia Lumia 635 mobile phone was used as DUT.

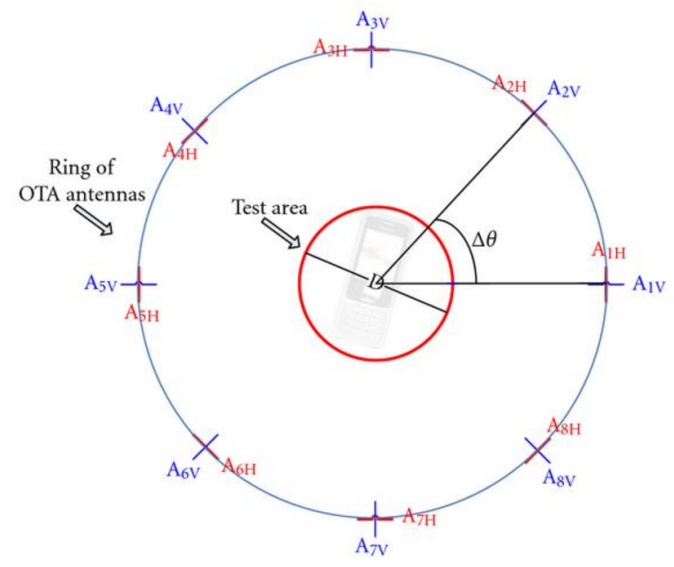

Fig. 1. OTA ring.

To emulate certain electromagnetic environment with certain spatial and temporal characteristics defined by the channel model, two techniques are used. One method is the prefaded signal synthesis (PFS), which has been implemented in commercial tools [9]-[11]. The other one is called planewave synthesis (PWS) [10], [12]-[16]. Both methods should be equally capable to emulate electromagnetic environment even though they use different channel generation methods. 


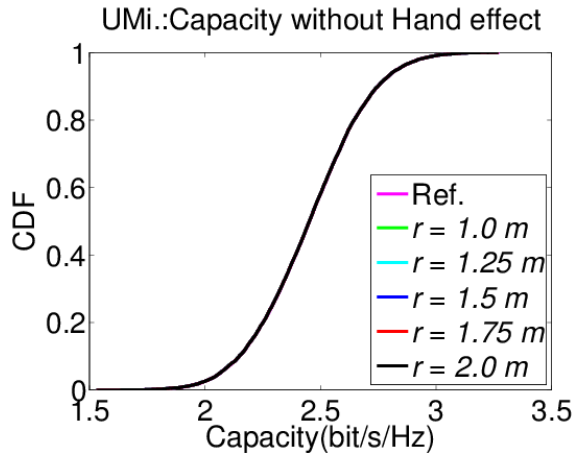

(a)

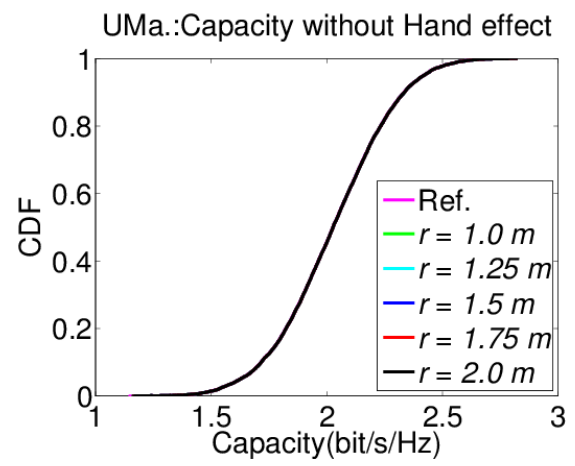

(c)

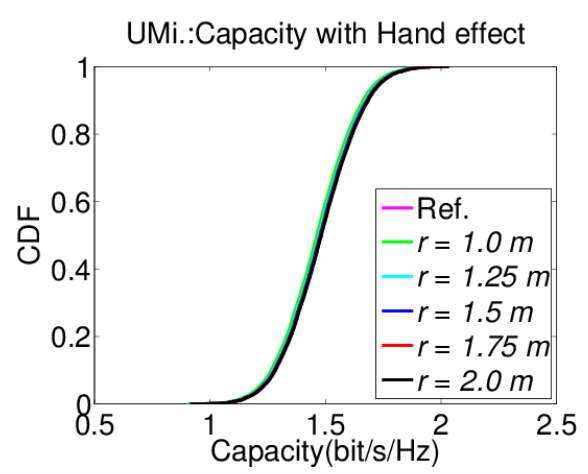

(b)

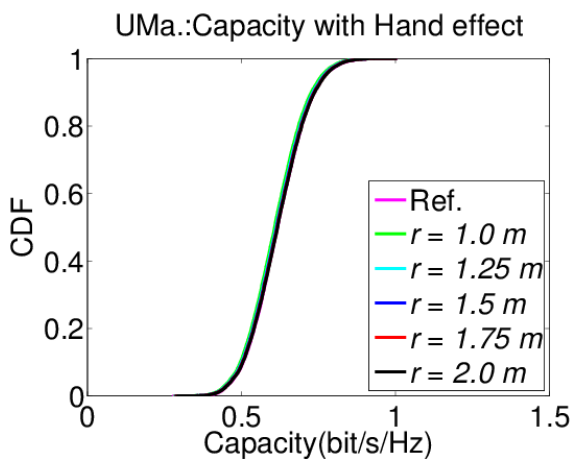

(d)

Fig. 2. Capacity CDF for different probe ring radius at $806 \mathrm{MHz}$ (a) UMi without hand effect, (b) UMi with hand effect, (c) UMa without hand effect, and (d) UMa with hand effect.

In present work OTA ring radius was studied for both PWS and PFS cases.

\section{A. OTA ring radius simulations}

Prior to validation measurements numerical computations were performed. Simulations consisted of two parts. At first, simulation verification was performed using cumulative distribution function (CDF) of channel capacity calculations for urban micro-celluar (UMi), urban macro-cellular (UMa) models. As a DUT in both these cases measured antenna patterns of Nokia Lumia 635 in free space and in phantom hand (emulates real hand) were used gaining in total 4 simulated cases for each DUT orientation and frequency. In the Fig. 2 there are capacity CDF calculations for different radii of $1 \mathrm{~m}, 1.25 \mathrm{~m}, 1.5 \mathrm{~m}, 1.75 \mathrm{~m}$ and $2 \mathrm{~m}$. The method of capacity CDF comparison is described in [17]. The second step of simulations was verification via another type of calculations - LTE-A system simulator. It emulates data propagation via radiomodule of a DUT and defined radio channel. Therefore in order to perform theoretical verification with help of throughput calculation for certain radio channel, LTE-A simulator requires pre-defined channel data using Spatial Channel Model (SCM) or SCM extended (SCME) methods. LTE-A simulator is based on the [18] requirements and transfer randomly created data via loop over defined signal-to-noise (SNR) ratios. Every loop splits data to transport blocks and consists of cyclic redundancy check
(CRC) information insertion, segmentation, turbo coding, rate matching, scrambling, modulation, antenna mapping, convolution with channel data, and detecting the data with following comparison to sent bits. Throughput is calculated using part of transport block with errors in all sent transport blocks. Simulator supports automatic repeat request (ARQ) feature as well as hybrid ARQ (HARQ). Channel end simulator parameters initialization is done once before the loop over SNRs since channel data except of SNR and simulation parameters such as quadrature amplitude modulation (QAM)

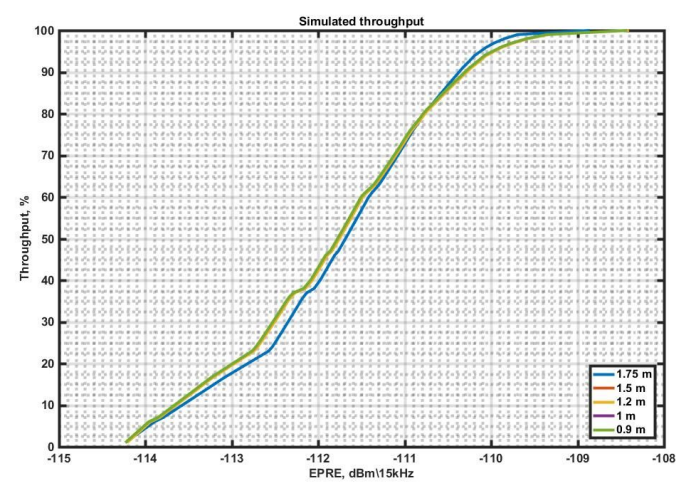

Fig. 3. Simulations for different OTA ring radii using LTE-A simulator. Frequency is $806 \mathrm{MHz}$, vertical DUT orientation, MIMO 2x2 connection, UMi. DUT rotation step is $45^{\circ}$. Some curves are covered by another. 
order or Modulation and Coding Scheme (MCS) index value stay the same. For simulation of radius dependence MCS was 20, HARQ number was 1, list sphere detector was used and list size was 64 , channel speed was $5 \mathrm{~km} / \mathrm{h}$ and bandwidth was $10 \mathrm{MHz}$.

LTE-A simulator developed in CWC was used for the simulation of the DUT behavior in different OTA ring radii keeping all other parameters fixed. Channels used in capacity CDF validation was used in LTE-A simulator and results are presented in the Fig. 3.

Both types of simulations do not show dependence from OTA ring radius and give the estimation of possible chamber sizes that can be used for validation and certification measurements.

\section{B. Throughput measurements for different OTA ring radii}

Measurements were performed in the anechoic chamber of Centre for Wireless Communications (CWC). Throughput was chosen as a figure of merit for the effect of OTA ring size (value of relative throughput at certain transmit power). Radius dependence was verified using the throughput measurement for each radius. Radii set was chosen to be $1 \mathrm{~m}$, $1.2 \mathrm{~m}, 1.5 \mathrm{~m}, 1.9 \mathrm{~m}, 2 \mathrm{~m}$. Minimal radius of $0.9 \mathrm{~m}$ with certain restrictions was tested in ability to provide reliable result. Smaller radii are not supported in current OTA ring configuration in the anechoic chamber of CWC.

Throughput radius dependence was measured for the PFS case and than the same measurements were repeated for the case of PWS.

Throughput curves for different radii that are presented in the Figs. 4(a) to 4(d) depict PFS case. Measured energy per resource element (EPRE) values at $70 \%$ throughput are presented in the upper part of Table I for comparison.

TABLE I

RADIUS DEPENDENCE MEASURED IN CWC ANECHOIC CHAMBER. EPRE VALUES MEASURED AT $70 \%$ THROUGHPUT WITH DIFFERENT RADII, PFS AND PWS.

\begin{tabular}{|l|c|c|c|c|c|c|}
\hline \hline Radius & $1 \mathrm{~m}$ & $1.2 \mathrm{~m}$ & $1.5 \mathrm{~m}$ & $1.75 \mathrm{~m}$ & $1.9 \mathrm{~m}$ & $2 \mathrm{~m}$ \\
\hline & \multicolumn{7}{|c|}{ PFS, EPRE, $(\mathrm{dBm} / 15 \mathrm{kHz})$} \\
\hline UMa, 806 MHz & -93.1 & -94 & - & & & -95.1 \\
\hline UMa, 2655 MHz & -102.3 & -102.3 & -103.3 & & & 102.7 \\
\hline \hline UMi, 806 MHz & -101.6 & -101.6 & -103.1 & & & -103.1 \\
\hline $\mathrm{UMi}, 2655 \mathrm{MHz}$ & -109.3 & -109.5 & -109.7 & & & -109.7 \\
\hline \hline & \multicolumn{7}{|c|}{ PWS, EPRE, (dBm/15kHz) } \\
\hline UMi, 806 MHz & -108.8 & -108.8 & -111.6 & -112.38 & -112.4 & -112.41 \\
\hline UMa, 806 MHz & -101 & -103 & -101.8 & -102.8 & -102.4 & -102.8 \\
\hline
\end{tabular}

In Figs. 5(a) and 5(b) results for PWS are shown. Bottom of the Table I summarizes findings for radius dependence in PWS. In comparison to PFS clear trend of moving to higher EPRE values can be observed for PWS UMi with decrease OTA of ring radius.

\section{DISCUSSION}

Simulations using capacity CDF calculations have been performed to obtain the minimum distance between probe antennas and DUT. The minimum distance has been derived against channel capacity. Fig. 2 shows the capacity CDFs with different probe ring radius $r_{\text {OTA }}$ for DUTs with and without hand. As the distance $r_{\text {OTA }}$ less than $1 \mathrm{~m}$ does not fulfill the far-field requirement defined in [19], distances less than $1 \mathrm{~m}$ were not considered in the simulation. For the both scenarios, simulated capacity CDFs with $r_{\text {OTA }}$ from $1 \mathrm{~m}$ to $2 \mathrm{~m}$ are very close to the reference [18], which directly comes from SCME channel model. Simulated results show $r_{\text {OTA }}=1 \mathrm{~m}$ as optimal OTA ring radius size from economy point of view and this result is independent from scenario and hand effect. Such findings are confirmed for the case of measurements using PFS technique. For the case of PWS UMi there is a correlation between OTA ring radius and obtained EPRE values at the corresponding throughput values.

Simulation results using LTE-A simulator are shown in Fig. 3 and they also have very good correspondence to measurements in case of PFS technique. Similarly to capacity CDF calculations they are not any more accurate for the case of PWS (Fig. 5). Both types of simulators show equal capability in predicting of PFS technique based measurements. Therefore, capacity CDF calculation and LTE-A simulator were verified as equal simulation methods that provide adequate simulations for PFS.

For the possible explanation of such behavior in case of PWS UMi correlation between far field and near field criteria can be studied. Taking into account position of the phase center of Vivaldi antenna [20], [21] OTA ring radius should be recalculated for the values of distances between the phase centers of DUT and probe antennas which are presented in the Table II. Phase center can be estimate to be in the closest to ring center Vivaldi antenna edge. It means that OTA ring radius measured from the center to the antenna mounting point should be corrected to the length of Vivaldi antenna used in the measurements. Thus trend observed in measurements for radii less than $1.75 \mathrm{~m}$ (shown in the Figs. 5(a) and 5(b)) can be explained by the brake of the far field approach.

TABLE II

EFFECTIVE RADIUS OF THE OTA RING (MEASURED FROM THE PHASE CENTER OF PROBE ANTENNA)

\begin{tabular}{|l|c|c|c|c|c|}
\hline Radius & $1 \mathrm{~m}$ & $1.2 \mathrm{~m}$ & $1.5 \mathrm{~m}$ & $1.75 \mathrm{~m}$ & $2 \mathrm{~m}$ \\
\hline Effect. Radius & $0.75 \mathrm{~m}$ & $0.95 \mathrm{~m}$ & $1.25 \mathrm{~m}$ & $1.5 \mathrm{~m}$ & $1.75 \mathrm{~m}$ \\
\hline
\end{tabular}

It means that throughput and power values form the Fig. 5(a) show the difference between far- and nearfield zones for OTA measurements for the case of UMi at 806 $\mathrm{MHz}$ PWS. Fig. 5(b) depict the same trend for scenario UMa.

\section{CONCLUSION}

Performed simulations did not show any OTA ring radius dependence while in measurements for the case of PWS such trend was visible. Measurements using PWS technique shown qualitative difference in obtained results. Radii of $1.7 \mathrm{~m}$ and bigger show absence of radius dependence, radii 


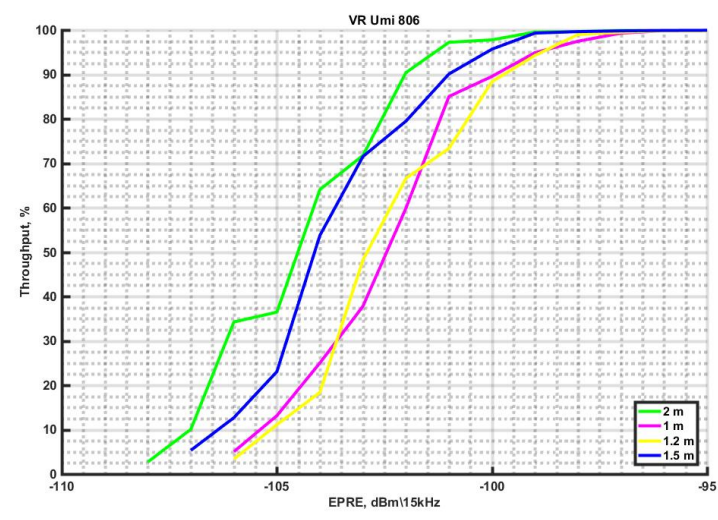

(a)

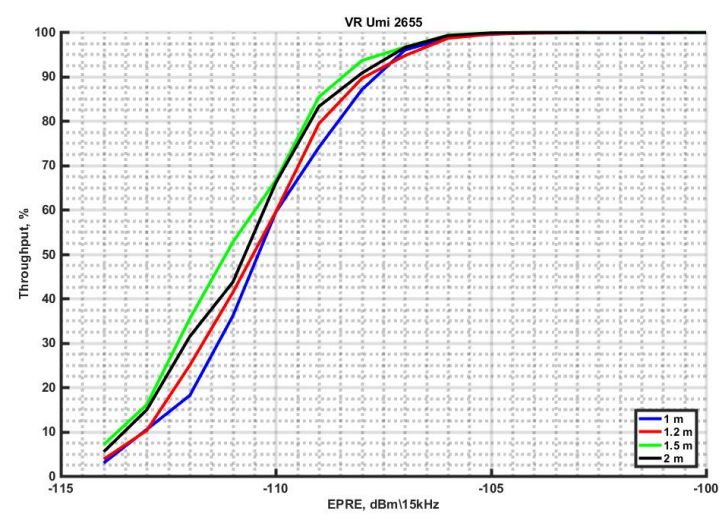

(c)

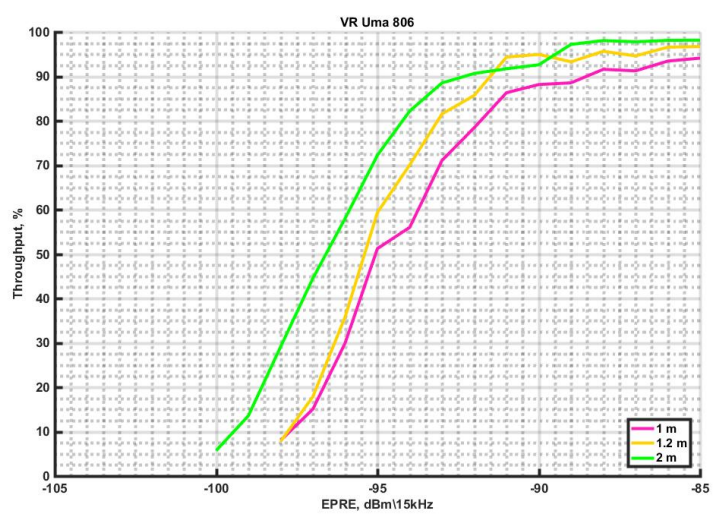

(b)

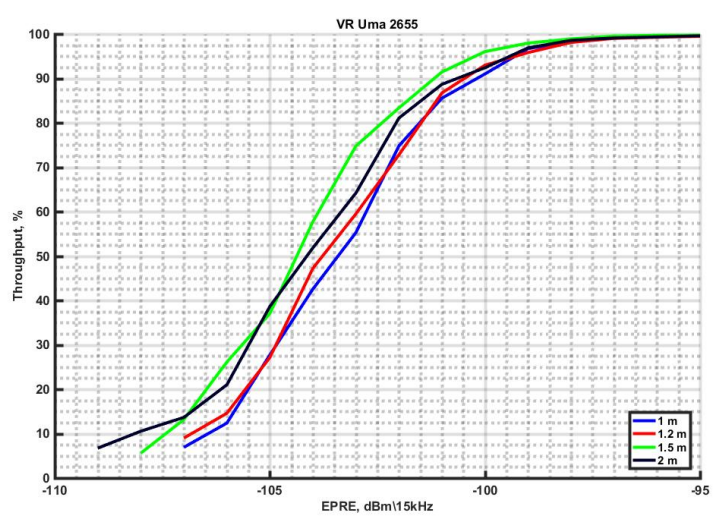

(d)

Fig. 4. Measured throughput of Nokia Lumia 635 with MIMO $2 \times 2$ reference antennas, PFS. Phone orientation is P0. (a) UMi, frequency is $806 \mathrm{MHz}$, (b) UMa, frequency is $806 \mathrm{MHz}$, (c) UMi, frequency is $2655 \mathrm{MHz}$, (d) UMa, frequency is $2655 \mathrm{MHz}$.

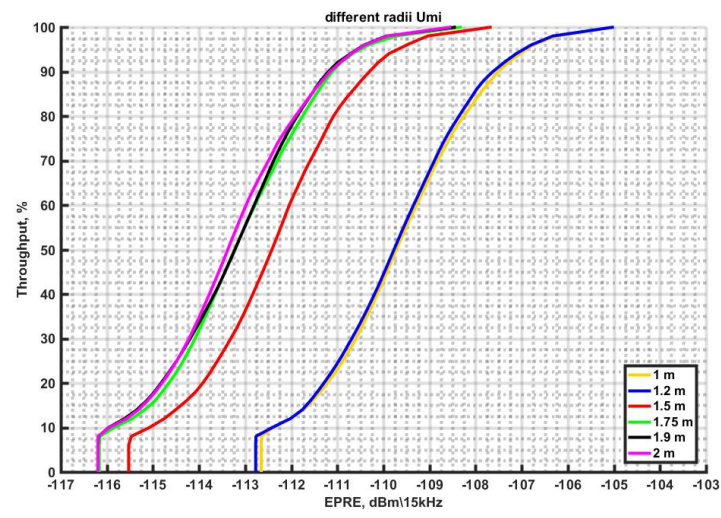

(a)

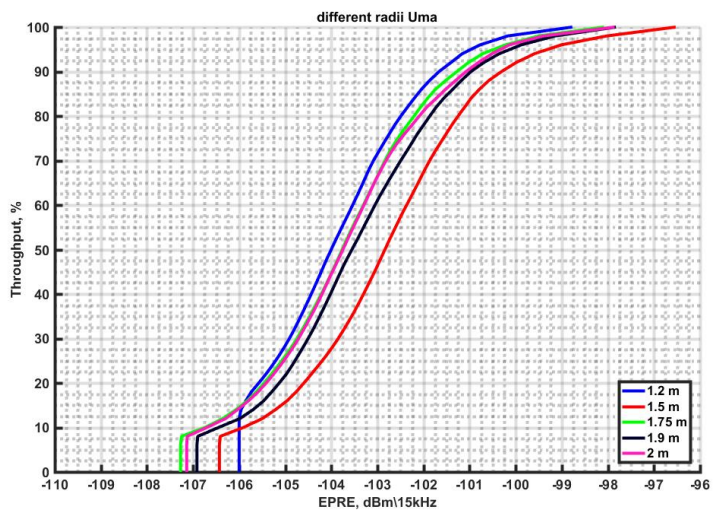

(b)

Fig. 5. Measured throughput of Nokia Lumia 635 with MIMO 2x2 reference antennas, PWS. Phone orientation is P0. (a) UMi, frequency is $806 \mathrm{MHz}$, (b) UMa, frequency is $806 \mathrm{MHz}$.

smaller than $1.2 \mathrm{~m}$ also show radius independence and these two cases have EPRE difference of about $4 \mathrm{~dB}$. Middle radius of $1.5 \mathrm{~m}$ is between these two different radii zones. Explanation was proposed for description of the effect of OTA ring size to measured throughput value. Tables I and II lead towards the conclusion that PWS is sensitive to stability of plane waves which is practically hard to control in OTA rings smaller than $1.5 \mathrm{~m}$ due to breakage of far field condition. 


\section{ACKNOWLEDGMENT}

The authors would like to thank to all the participants of this research. This project was supported by Tekes, Anite telecom, Nokia and ETS-Lindgren and authors thank for giving possibility to perform this research and for providing infrastructural facilities.

\section{REFERENCES}

[1] P. Kyösti and T. Laitinen. Over-the-air test, May 1 2014. US Patent App. 13/259,505.

[2] T. Laitinen, P. Kyösti, J. P. Nuutinen, and P. Vainikainen. On the number of ota antenna elements for plane-wave synthesis in a mimo-ota test system involving a circular antenna array. In Proceedings of the Fourth European Conference on Antennas and Propagation, pages 1-5, April 2010.

[3] P. Kyösti, J.P. Nuutinen, and T. Jämsä. Over-the air test, February 10 2015. US Patent 8,954,014.

[4] Verification of radiated multi-antenna reception performance of user equipment. In 3GPP, TR 37.977 V12.1.0, March 2014.

[5] B. Furht and S.A. Ahson. Long Term Evolution: 3GPP LTE Radio and Cellular Technology. Internet and Communications. CRC Press, 2016.

[6] N. Serafimov, P. S. Kildal, and T. Bolin. Comparison between radiation efficiencies of phone antennas and radiated power of mobile phones measured in anechoic chambers and reverberation chamber. In IEEE Antennas and Propagation Society International Symposium (IEEE Cat. No.02CH37313), volume 2, pages 478-481 vol.2, 2002.

[7] P. Kyösti and L. Hentilä. Criteria for physical dimensions of mimo ota multi-probe test setup. In 2012 6th European Conference on Antennas and Propagation (EUCAP), pages 2055-2059, March 2012.

[8] Atsushi Yamamoto, T Sakata, T Hayashi, K Ogawa, K Sakaguchi, and JI Takada. Procedure of designing the structural parameters of a spatial fading emulator with a laplacian angular power spectrum of incoming wave. COST2100, 2010.

[9] W. Fan, X. Carreo, J. Nielsen, J. S. Ashta, G. F. Pedersen, and M. B. Knudsen. Verification of emulated channels in multi-probe based mimo ota testing setup. In Proc. 7th European Conf. Ant. Prop. (EuCAP 2013), pages 97-101, Gothenburg, Sweden, 2013.

[10] P. Kyösti, T. Jämsä, and J. Nuutinen. Channel modelling for multiprobe over-the-air MIMO testing. Int. J. Ant. Prop., 2012.

[11] W. Fan. Over the Air Testing of MIMO Capable Terminals. PhD thesis, Aalborg University, Aalborg, Denmark, Oct. 2014.

[12] T. Laitinen, P. Kyösti, J.-P. Nuutinen, and P. Vainikainen. On the number of OTA antenna elements for plane-wave synthesis in a MIMO-OTA test system involving a circular antenna array. In Proc. 4th European Conf. Ant. Prop. (EuCAP 2010), pages 1-5, Apr. 2010.

[13] J.T. Toivanen, T.A. Laitinen, V. Kolmonen, and P. Vainikainen. Reproduction of arbitrary multipath environments in laboratory conditions. IEEE Trans. Instr. Meas., 60(1):275-281, Jan. 2011.

[14] W. Fan, X. Carreo, J. Nielsen, K. Olesen, M. B. Knudsen, and G. F. Pedersen. Measurement verification of plane wave synthesis technique based on multiprobe mimo-ota setup. In IEEE Vehicular Technology Conference (VTC2012-Fall), pages 1-5, Quebec City, Canada, 2012.

[15] M.S. Miah, A. Khatun, and K. Haneda. Feasibility study of emulating extended spatial channel models in a multi-probe mimo ota antenna test setup. In Progress In Electromagnetics Research Symposium Proceedings (PIERS), Prague, Czech Republic, July 2015.

[16] A. Khatun, V. Kolmonen, V. Hovinen, D. Parveg, M. Berg, K.I. Haneda, K. Nikoskinen, and E.T. Salonen. Experimental verification of a plane-wave field synthesis technique for mimo ota antenna testing. IEEE Transactions on Antennas and Propagation, 64(7):3141-3150, July. 2016.

[17] M. M. Suzan, A. Khatun, K. Haneda, L. Hentila, and A. Karilainen. Effects of user's hand on the measurement setup in mimo over-the-air testing. In 2016 10th European Conference on Antennas and Propagation (EuCAP), pages 1-5, April 2016.

[18] Verification of radiated multi-antenna reception performance of user equipment. In 3GPP, TR 37.977 V12.1.0, March 2014.

[19] User equipment (ue) / mobile station (ms) over the air (ota) antenna performance conformance testing. In 3GPP TS 34.114 V7.0.0, 2008-2009.

[20] Hai Liu and Motoyuki Sato. in situ measurement of pavement thickness and dielectric permittivity by $\{\mathrm{GPR}\}$ using an antenna array. $\{N D T\} \&$ E International, 64:65 - 71, 2014.

[21] Bo-shi Jin, Qun Wu, Yu-ming Wu, Li Bian, and LeWei Li. An Approach to the Determination of the Phase Center of Vivaldi-Based UWB Antenna, pages 69-73. Springer New York, New York, NY, 2007. 